\title{
Prefacio de las perspectivas del tiempo en el arte y la educación, por Adela Olmo Soto.
}

\author{
Adela Olmo Soto \\ Universidad de Córdoba
}

\section{RESUMEN}

Podríamos decir que el tiempo se ha detenido durante esta pandemia, dándonos así la posibilidad de percibir aspectos tanto intrínsecos como extrínsecos a nosotras, que tal vez, hasta este momento, pasaban inadvertidos. La individualidad que acompaña a la postmodernidad, implica experiencias y opiniones sustancialmente diferentes en este proceso de percepción. Es esta pluralidad la que late en los encuentros entre artistas y, a su vez, es la que nos conecta.

En los próximos capítulos escribo sobre las conferencias y debates desarrollados durante el 32 Encontro da APECV (Associação de Professores de Expressão e Comunicação Visual). Aunque a todas nos unía el concepto común del tiempo, la increíble variedad de estudios, propuestas y reflexiones nos obliga a plantear una estructura para navegar por la información, relacionando conceptos y planteando nuevas e innovadoras ideas.

PALABRAS CLAVE: Dibujo. Tiempo. Percepción. Enseñanza. Identidades. Relaciones.

\section{ABSTRACT}

We could say that time has stopped during this pandemic, thus giving us the possibility of perceiving both intrinsic and extrinsic aspects to us, which perhaps, until now, went unnoticed. The individuality that accompanies postmodernism implies substantially different experiences and opinions in this process of perception. It is this plurality that beats in the encounters between artists and, in turn, is what connects us.

In the next chapters I write about the conferences and debates developed during the 32nd Encontro da APECV (Associação de Professores de Expressão e Comunicação Visual). Although the common concept of time united us all, the incredible variety of studies, proposals and reflections forces us to propose a structure for navigating information, linking concepts and proposing new and innovative ideas.

KEYWORDS: Art. Education. Drawing. Time. Perception. Teaching. Identities. Relationships. 


\section{Capítulo 1. El tiempo en las artes.}

El juego sobre el tiempo guarda una relación intrínseca con el arte y la educación artística, llevándonos en estos momentos a enfrentarnos a otros modos de vivencia. EI intento de definirlos, por parte de Paulo Pires do Vale, le lleva a remitirse a la Grecia clásica, representando a través de ella el tiempo cualitativo, cuantitativo y eterno.

Cabe subrayar también, una división que nos ha acompañado e incluso atormentado durante estos días de confinamiento: la clasificación del tiempo entre productivo y no productivo. Al aceptar los ritmos de la creación resulta indispensable asumir, de igual manera, la necesidad de ralentizarlos en ocasiones e incluso congelarlos, abriéndonos a la reflexión y observación.

Como último punto de la comunicación se destaca el papel del artista, definiéndolo como un "niño que juega", es decir, alguien que experimenta e investiga con suma curiosidad y a menudo sin miedo. Opiniones ya expresabas por teóricos del arte como Jonh Berger, quien describe al dibujo en la niñez como "un juego inconsciente, un acto innato de comunicación mediante signos visuales", siendo el arte parte vital de su formación (Berger, 2011).

Marcelo Simon Wasem se muestra a favor de la desaceleración de las temporalidades como camino para la creación poética de las artes. El arte, al igual que el acto de crearlo, supone transformar los sentidos en materias. Nos encontramos con una experiencia discontinua y primitiva, en la que tenemos que estar en sintonía con nuestro yo interno.

De igual manera, no cabe duda de que el arte está conectado al territorio que habitamos y al contexto, formando parte de las distintas identidades individuales de una comunidad. Este además, puede plantearse como una forma de reivindicación en sí misma. Es posible reivindicar el propio arte mientras reivindicamos otras ideas a través de él.

Una de las grandes preocupaciones del siglo es y será el cambio climático. La reducción de la producción y la movilidad en estos tiempos de pandemia ha dejado entrever la belleza de la naturaleza, tan olvidada sobretodo en las grandes ciudades. En mi caso, he tomado consciencia de la calidad del aire que respirábamos al notar, las primeras semanas de crisis, su significativa mejoría.

El arte como herramienta provocadora y revolucionaria está hoy más presente que nunca. Artistas como Ai Weiwei o el anónimo Banksy son una prueba del poder y simbología de los productos artísticos. Como cabía esperar en nuestro encuentro, este perfil de creadores y creadoras también surge. Alejandro Navarro Lara nos narra su experiencia personal, nacida a través de la motivación de buscar un arte útil, que incite a mejorar el mundo. Como no, tomando conceptos de la ecología y en la regla de reducir, reutilizar y reciclar.
Se nos presenta un análisis del uso que le damos a los automóviles, tan sencillo como contar la cantidad de kilómetros que recorremos con él y para qué situaciones elegimos usarlo, llevándonos a la clara conclusión de que se hace un uso exagerado de los mismos. Nuestro autor nos invita a replantearnos la verdadera necesidad de este medio de transporte, poniendo sobre la mesa una realidad ya intuida, pero a menudo silenciada.

Por último en este apartado se destacará el papel de María Paz Barrios Mudarra, con su muestra del arte a través de la poesía narrativa, que aproxima lo visual y los sentimientos evocadores de la propia literatura. Con versos que hablan de las etapas de la vida, las palabras a través de las tecnologías y los abrazos virtuales.

Con estas afirmaciones se transmite la importancia de las tecnologías y el arte de las letras. Importancia destacada en estos tiempos ya que gracias a ellas hemos podido trascender como colectivo y sociedad durante la presente crisis sanitaria.

El mensaje de bondad resuena en lo más profundo, si necesitas transmitir bellos sentimientos a alguien, hazlo, eligiendo las herramientas y medios que prefieras, ya sean dibujos, palabras, música o incluso fotografías, pero nunca te lo guardes, porque con un pequeño detalle puedes alegrar un día. El propósito último del tiempo, es encender una luz en la oscuridad.

\section{Capítulo 2. Planteamientos y tiempos de la educación artística.}

Ana Sousa muestra a la pedagogía de las artes como una mezcla entre ensayos didácticos y empíricos. Como un aprendizaje construido a través de experiencias que transforman nuestra vida y que a su vez ayudan a construir la identidad. Experiencias que, debido a la dicotomía entre distanciamiento y cercanía que provoca internet en estos tiempos, presentan otro formato o estructura. Situación que nos motiva a implementar una nueva metodología en la educación.

La forma de impartir una clase irá siempre en relación con el contexto personal del propio docente, como nos comentó Marcelo Forte. Y dentro de esto es fundamental también el papel de las redes sociales. Las dimensiones socioculturales de la vida contemporánea, llena de dinamismo, terminan estableciendo una curiosa relación entre la identidad a través de la proyección de nuestra persona en retratos de terceros, buscando similitudes en ellos.

Así se muestra en la ponencia sobre la investigación del profesor José Rubilar Medina, quien a través de ella nos muestra lo llamativo del fenómeno del selfie, entre procesos subjetivos y plurales, planteando interrogantes en torno a los procesos identitarios, tanto a nivel individual como colectivo. 
En este encuentro entre profesores de artes plásticas, han sido muchas las alusiones a métodos de enseñanza en los grados universitarios encargados de formar a maestros. Dentro de esta didáctica de las artes plásticas, encargada en esta ocasión de formar a adultos para estos a su vez formar a jóvenes, nos encontramos a María Lorena Cueva Ramírez.

Ella comienza su ponencia recordándonos la opinión general de la interrelación entre el trabajo manual y las artes plásticas, y la triste dinámica que a menudo lo relaciona casi en exclusiva con manualidades. Una relación que, como artistas, resulta totalmente obsoleta al contar hoy día con tantas plataformas y medios para la creación.

También nos plantea la experiencia de un taller para el alumnado del Grado de Educación Infantil, donde el collage supone el punto de partida. Elegido por sus posibilidades expresivas, que permiten integrar elementos de la realidad en la obra, existiendo en ellos diferentes tiempos. A través de este tipo de proyectos se liga el material a conceptos, situaciones y vivencias, por lo que tiene un alto componente abstracto.

Tal vez estos términos no sean trabajados directamente a edades tempranas. Como sabemos, la educación artística depende de las experiencias del propio profesor o profesora, así que, partiendo de la interrelación entre todos estos conceptos, sería muy interesante ampliar las enseñanzas en este grado incluyendo los aspectos abstractos y conceptuales del arte, relacionándolos con temas actuales.

Según Inés López Manrique, una de las cuestiones primordiales de la educación es la motivación, tanto para el docente a la hora de desempeñar su labor como para el alumnado. Existen muchas formas de motivar y motivarse, aunque existe un factor fundamental: la relación con los resultados académicos y el autoconcepto.

El tiempo no suele tenerse en cuenta para el factor motivacional, pero este puede ayudar a alcanzarlo. Como bien sabemos cada individuo tiene unos ritmos, fundamentales para su desarrollo. Seguramente este sistema educativo, que marca una pautas y plazos un tanto rígidas, atenta contra esta necesidad de desarrollo dentro de unos plazos personales.

Es parte de la labor de los profesores reconocer y valorar los tiempos, tanto los propios como los de sus alumnos y alumnas. Destacando la necesidad de frenar para fomentar el autocuidado y mejorar el concepto que tenemos de nosotras mismas como seres únicos.

Alfredo Betâmio de Almeida era un artista y profesor portugués desconocido para mí hasta ahora. Ha sido a través de Elisabete Oliveira que hemos podido recuperar y profundizar un poco más de su obra en este encuentro. Nuestro artista mantuvo una significativa actividad, destacando por el uso de pintura a pastel, acuarela y gouache, con los temas predominantes de bodegones y pintura abstracta.
Nuestra compañera habló de tres aspectos desde los que estudiar la obra del autor, extensibles, en cierto modo, al análisis de la obra de la mayoría de artistas. Estas perspectivas se catalogan: en la material, relativa a las técnicas y herramientas que componen el cuerpo de la obra; la social, que depende de cómo afectó y fue afectada por su contexto; y por último, la ontológica, la que pretende responder a las cuestiones que plantea un trabajo artístico, buscándole la razón de ser a la obra.

Otra faceta que me llamó la atención de su trabajo fue la del intento de definir los perfiles de los profesores de dibujo de secundaria, valorando la atmósfera creativa que el profesor de dibujo podía aportar a sus clases. Para Betâmio de Almeida, el maestro debe ser el que estimule las necesidades expresivas del niño o adolescente.

María Laura Martín Moreno, especializada en el campo de la música, nos enseña el taller que imparte a niños de aproximadamente seis años, donde aplica la teoría de la personalidad de Gordon. Trabaja la observación y la escucha de manera dinámica, valiéndose a menudo del canto y poniendo a las emociones como estructura central de sus enseñanzas.

Durante muchos años la educación tradicional nos ha enseñado sistemáticamente a reproducir patrones de comportamiento asépticos, en los que los sentimientos quedan desplazados. Como sabemos las artes guardan una íntima relación con las emociones, por lo que cambiar la dinámica de las clases atendiendo al valor de las emociones y la sintonía entre ellas es tanto reformador, como subversivo.

\section{Capítulo 3. La formación para la educación artística.}

Aunque el aprendizaje artístico es vital, lamentablemente se ha visto degradado en la educación obligatoria, no manteniéndose en muchos casos, al elegir imponer materias que se entienden como más relevantes para la futura vida profesional productiva. Estas son las relativas a la economía capitalista, cuya viabilidad parece cuestionarse más que nunca en estos momentos.

Mi ponencia en el congreso se fundamentó en esta línea de investigación. Debido a mis inquietudes y mi formación, tanto como artista en la Facultad de Bellas Artes de Sevilla y a la vez como profesora de dibujo, decidí enfocar la investigación en torno a un análisis de las enseñanzas relativas a las artes plásticas. Compartiendo en estos momentos perspectivas temporales tanto de alumna, como de profesora.

La educación artística está presente en el sistema educativo, pero con una carga lectiva muy inferior a la necesaria $y, a$ veces también, con una distribución horaria poco adecuada, llegando a quedar relegada a ojos de la sociedad como una asignatura fácil de aprobar y con nula o escasa importancia. Esto termina degenerando en una carencia educativa 
de la que poco se ha estudiado, aunque es fácil intuir las consecuencias, sobre todo para las personas que han tenido formación artística de mayor profundidad.

Por lo tanto, enfoqué mi investigación en la formación de artes plásticas, que se enseña a quienes en el futuro darán clases a los niños y niñas, es decir las maestras/os. Me remitiré al primer momento, o tiempo, que termina generando este concepto negativo de la educación artística, haciendo para ello un análisis de los Grados de Educación Infantil y Educación Primaria impartidos en la Universidad de Córdoba, España.

Partiendo de un planteamiento objetivo, me baso en la mutable y a menudo poco conforme legislación vigente, y la relaciono con las guías docentes de las asignaturas relativas a las artes plásticas, donde se muestra los conceptos y técnicas trabajadas, así como el número total de horas y créditos ECTS.

A través de ellas es fácil determinar la cantidad y la calidad de las asignaturas que se imparte a los futuros maestros. Ya que parte de su labor será transmitir conocimientos básicos en el campo de las artes, los cuales supondrán la base donde profundizar en tiempos posteriores. Las asignaturas impartidas, relativas a las artes plásticas, aparecen en la siguiente tabla:

\begin{tabular}{|c|c|c|c|c|c|}
\hline Nombre asignatura & Titulación & Tipología & $\begin{array}{c}\mathbf{N}^{\circ} \text { de } \\
\text { créditos }\end{array}$ & Curso & Horas \\
\hline $\begin{array}{c}\text { Expresión Plástica infantil } \\
\text { y su didáctica }\end{array}$ & $\begin{array}{c}\text { Grado en Educación } \\
\text { Infantil }\end{array}$ & Obligatoria & 9 & $2^{\circ}$ & 225 \\
\hline $\begin{array}{c}\text { Patrimonio Histórico- } \\
\text { artístico y escuela }\end{array}$ & $\begin{array}{c}\text { Grado en Educación } \\
\text { Infantil }\end{array}$ & Optativa & 6 & $4^{\circ}$ & 150 \\
\hline $\begin{array}{c}\text { Artesanía Contemporánea } \\
\text { y la Educación Infantil }\end{array}$ & $\begin{array}{c}\text { Grado en Educación } \\
\text { Infantil }\end{array}$ & Optativa & 6 & $4^{\circ}$ & 150 \\
\hline Educación Plástica y \\
Visual
\end{tabular}

Tabla 1. Asignaturas sobre artes plásticas. Creación propia.

Como vemos son tres del Grado en Educación Infantil, dos optativas y una obligatoria, que suman un total de 21 créditos. Y solamente una del Grado en Educación Primaria titulada Educación Plástica y Visual, esta sí es obligatoria, y supone 6 créditos.

Ya que este proyecto estaba planteado para estudiar los planes de estudio de muchas universidades, me ha ayudado de un programa llamado QDA MINER que sirve para analizar categorías y frecuencias. $Y$ este ha generado los siguientes diagramas de barras donde se muestra la puntuación obtenida en las diferentes habilidades que engloban la formación artística, según la legislación vigente.

Los resultados son estremecedores, cuanto menos. En el caso del Grado en Educación Infantil podemos decir que alcanza los objetivos, aunque dos de las tres asignaturas son de carácter optativo. Para proporcionar un aprendizaje más completo sería necesario el cambio de su naturaleza a obligatorio.

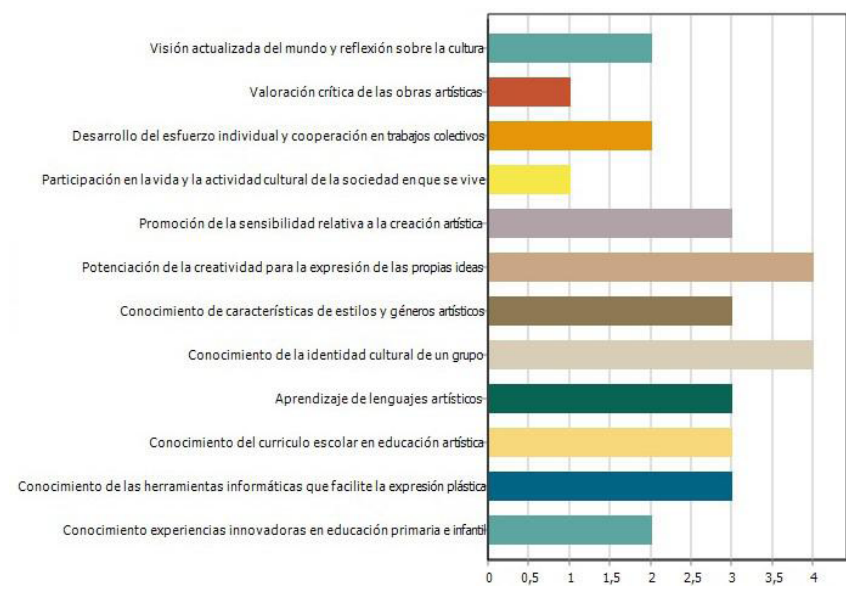

Figura 1. Diagrama de frecuencias del Grado de Educación Infantil. QDA Miner.

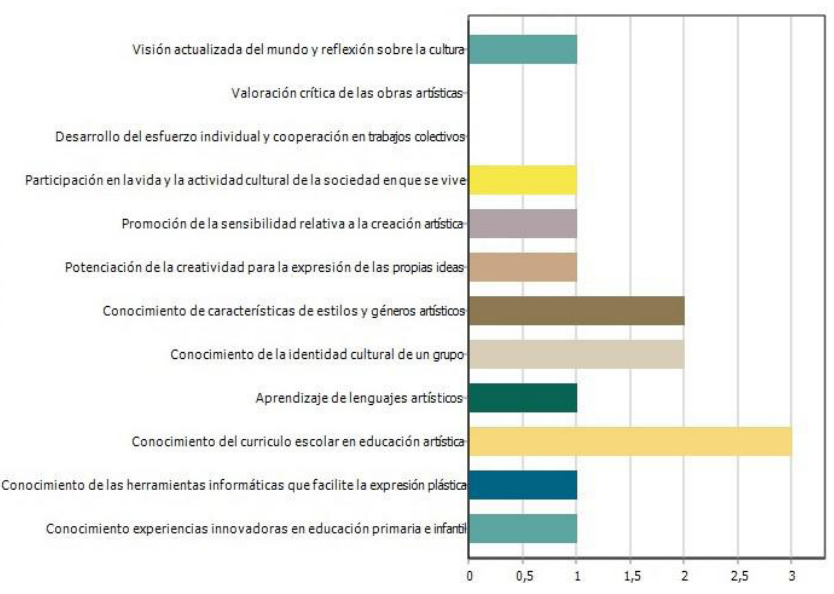

Figura 2. Diagrama de frecuencias del Grado de Educación Primaria. QDA Miner.

Por su lado, la asignatura objeto de estudio en el Grado en Educación Primaria, resulta del todo insuficiente para alcanzar la totalidad de acciones que recoge la legislación en la competencia referida.

6 créditos ECTS, de los 240 que componen el grado, suponen muy poca carga lectiva. Sería necesario implementar nuevas asignaturas dentro del plan de estudios en la Universidad de Córdoba, dando cobertura a las acciones necesarias para su obtención.

Esta situación confirma la idea, previamente intuida, de que se ha producido una disminución muy acusada de las materias sociológicas y psicopedagógicas, en función de un mayor contenido científico en los planes de estudio de estos grados.

Esto a su vez nos lleva a reflexionar acerca de la importancia de que la legislación educativa englobe las artes, configurándose en base a los nuevos métodos para la educación y adaptándose, de esta manera, a la situación social y los tiempos en los que vivimos. La facilidad para la comunicación ha logrado que, muchas de estas experiencias y métodos sean mostrados entre las personalidades que hemos coincidido en este encuentro. 


\section{Capítulo 4. Reflexiones finales, el arte y la educación en} nuestros tiempos.

La reflexión acerca de los temas tratados lleva a plantear una notoria sinergia entre tiempo y arte. No nos remitimos en exclusiva al componente temporal de cualquier acción, al desarrollarse esta en un momento determinado, sino que nos lleva, en su visión más romántica, a una comparación entre la existencia del tiempo a la vez que el arte.

En el transcurso de las últimas décadas nos hemos encontrado con el sistemático interés de apartarnos de nuestro lado más empático, emocional y social, atributos íntimamente relacionados con las artes y humanidades. Esta insistencia aparece reflejada en la educación artística, al relegarla en el proceso educativo a un segundo plano. Aunque en sus líneas nos encontramos un sinfín de aspectos de gran riqueza, cuya importancia es vital para nuestro desarrollo.

No obstante, aun habiéndose producido este acusado descenso de las nociones artísticas en la educación, la realidad con la que nos encontramos es que este no desaparece de la sociedad. Generalmente, el arte es motivado por inquietudes e intereses, tanto individuales como colectivos, pero en otras ocasiones nos encontramos en las manifestaciones artísticas una simple y pura necesidad de existir. Al igual que el tiempo transcurre inevitablemente, el arte ocurre de la misma manera.

Al igual que las manifestaciones artísticas, se podría decir que los tiempos en los que vivimos se han vuelto relativos: no existe una verdad universal ni objetiva. La información fluye a través de la red a un ritmo vertiginoso, volviéndose en ocasiones muy difícil diferenciar la que se remite a datos y situaciones reales de la que no. Tal vez todo merece consideración, pero definitivamente no todo puede ser válido.

Esta situación, acentuada con la crisis sanitaria vivida y la obligatoria necesidad de permanecer aislados físicamente, nos ha obligado a reflexionar acerca del sistema al que estábamos sometidos y dábamos por válido, planteando la necesidad de reformular la mayoría de sus aspectos.

Ciñéndonos al enfoque relativo a las artes podemos decir que por primera vez, de forma generalizada, se ha mostrado de forma significativa la importancia de estas, tanto a título individual, buscando el autoconocimiento, como colectivo, una forma de vínculo y pertenencia a un grupo.

Sobra nombrar las reuniones desde los balcones, acompañadas de música, risas, y aplausos, donde a su vez los más jóvenes han mostrado sus dibujos de arcoíris y esperanza que han sido tan comunes durante el confinamiento.

De igual manera, internet se ha convertido durante estos días en el nuevo espacio expositivo por excelencia, al compartir con los balcones esa posibilidad de conexión con el exterior. De forma en absoluto sorprendente, el medio usado para conectar ha sido a través del arte. Quién podría imaginar una cuarentena sin la posibilidad de expresar con la creación, o encontrándola y viajando a través de ella, con libros, música, series o películas.

Como se ha demostrado en estas semanas, el arte y sus expresiones es un ingrediente que el ser humano necesita de forma innata. Aunque ya eran conocidos muchos estudios que destacan la importancia de la educación artística, al ser esta necesaria para potenciar la resolución de problemas, la memoria y la capacidad de aprendizaje en los niños y niñas, mejorando incluso la atención y el comportamiento.

Muchas de las formas de expresión artística se han venido desarrollado dentro de lo que conocemos como cultura visual. Su papel se ve especialmente potenciado gracias a las nuevas tecnologías, no sólo en el ámbito de la creación, sino también para su distribución, que es cada vez mayor. La cultura visual estudia la importancia de la imagen en las sociedades contemporáneas, ya que esta influye decisivamente en nuestro modo de vivir y pensar.

Como artistas y docentes, nuestra función no es solamente educar y enseñar a las futuras generaciones, sino que también recae la responsabilidad de ejercer la presión necesaria para evitar que con cada reforma educativa queden cada vez más relegadas, precisamente las asignaturas que fomentan el pensamiento crítico, la cultura y la humanidad. Nos encontramos en unos momentos complejos, que han puesto de manifiesto la obligatoriedad de cambiar y es necesario instigar a ello.

\section{Referencias.}

John Berger. (2011). Sobre el dibujo. España: Editorial Gustavo Gili. 